\title{
Review: morphine, eutectic mixture of local anaesthetics, ketorolac, and sucrose reduce postoperative and procedural pain in children
}

\author{
Maikler VE. Pharmacologic pain management in children: a review of intervention research.J Pediatr Nurs 1998 Feb;13:3-14.
}

\section{Question}

Which pharmacological interventions are effective for managing acute postoperative or procedural pain in children?

\section{Data sources}

Studies from 1987-96 were identified by searching Medline, CINAHL, CancerLit, and AIDSLINE using combinations of search terms including pain, analgesia, anesthetics, opioids, and age related terms (eg, neonate and toddler). Bibliographies of relevant articles were also reviewed.

\section{Study selection}

Studies were included if they tested a pharmacological intervention using a prospective design in a sample of $\geqslant 30$ people $<20$ years of age who were randomly allocated to $\geqslant 2$ groups. Studies had to provide adequate methodological and statistical details for review.

\section{Data extraction}

Data were extracted on type of pain, age of children, study design, type of intervention, outcomes and measurement approaches, and results.

\section{Main results}

41 studies of acute pain met the inclusion criteria. 22 studies focused on postoperative pain and 19 focused on pain associated with procedures (venipuncture, circumcision, suturing, heelsticks, immunisations, port wine stain removal, and intravenous [IV] port access). Study sample size ranged from 30-467 patients. Outcomes included self reported pain, observed pain behaviour, physiological changes, and analgesic consumption.

28 studies tested the efficacy of specific analgesic agents (including morphine, eutectic mixture of local anaesthetics [EMLA], ketorolac, lidocaine, and sucrose). 5 studies reported that EMLA was better than placebo for procedural pain and 2 studies found no differences between cream and patch formula- tions. Ketorolac resulted in lower pain scores and lower analgesic use compared with placebo (3 studies) or acetaminophen (1 study), but was as effective as morphine in 1 study and less effective than morphine in another study.

2 studies found that during circumcision, lidocaine cream resulted in less distress than placebo, but that lignocaine gel was less effective than penile block for pain and analgesic use in older children. In 3 studies, infants undergoing procedures who received sucrose (at concentrations $\geqslant 12 \%$ ) spent less time crying than those who received water or no treatment.

4 studies examined the effectiveness of different routes of administration (patient controlled analgesia [PCA], intramuscular, epidural, and continuous subcutaneous). Children preferred IV and PCA over intramuscular administration, and 2 studies found no differences in pain scores for PCA compared with epidural or subcutaneous administration. In 2 of 3 studies, however, PCA was associated with higher overall analgesic use.

3 studies tested the effectiveness of analgesics given prophylactically at the beginning of surgery and all reported lower postoperative pain scores and lower analgesic use in treatment groups than in placebo or no treatment groups. 5 studies examined the effects of the timing of postoperative IV morphine administration, comparing continuous administration, intermittent administration on demand (PCA), and intermittent plus continuous administration (PCA plus). No differences were found for PCA and PCA plus.

\section{Conclusions}

Morphine, eutectic mixture of local anaesthetics, ketorolac, and sucrose reduce postoperative and procedural pain in children. Intramuscular administration and patient controlled analgesia are less favourable in terms of patient satisfaction and total analgesic use, respectively.

Source of funding: no external funding.

For correspondence:Dr V Maikler, 600 South Paulina, Suite 1022, Chicago, IL 60612, USA.Fax +1 3129422549 .

\section{Commentary}

Few systematic reviews have evaluated the quality and quantity of research on the effectiveness of pharmacological interventions for managing acute pain in children. The study by Maikler evaluated several pharmacological interventions for relieving postoperative pain and pain associated with procedures.

This review used well defined inclusion criteria. Important characteristics of the study methodologies and findings were reported. However, data were not discussed on the numbers of children assigned to experimental, control, and placebo groups; specific outcome data (ie, means, medians, standard deviations, and ranges); and the statistical significance of the results. This lack of detail about the original studies makes it difficult for the reader to interpret the results of the review.
The quality of individual research studies was not discussed. In particular, issues surrounding the large variance in measurement strategies and types of interventions for pain relief were not addressed. This inconsistency in design, methods, and outcomes is usually the major reason why a meta-analysis cannot be done or can only be done with a small proportion of the studies conducted in a focused area. The author could have also included a more in depth analysis of the pain relieving interventions, particularly those using morphine, and provided rationale for why studies evaluating sucrose (which is usually considered nonpharmacological) were included with the pharmacological interventions.

This review supports the efficacy of several pharmacological interventions for children with acute pain, and provides some guidance in choosing the most effective and acceptable administration routes and timing of interventions. More work, however, needs to be done to develop more rigorous studies that will conclusively define which interventions nurses should use to best manage acute and chronic pain in infants and children, and particularly when to use nonpharmacological interventions such as sucrose.

Bonnie Stevens, RN, PhD Associate Professor Faculty of Nursing University of Toronto Career Scientist Ontario Ministry of Health Toronto, Ontario, Canada 